\title{
Scaling and further tests of heavy meson decay constant determinations from nonrelativistic QCD
}

\author{
S. Collins, ${ }^{1, *}$ C. T. H. Davies, ${ }^{1, *}$ J. Hein, ${ }^{2}$ G. P. Lepage, ${ }^{2}$ C. J. Morningstar, ${ }^{3}$ J. Shigemitsu, ${ }^{4}$ and J. Sloan ${ }^{5, \dagger}$ \\ ${ }^{1}$ Department of Physics \& Astronomy, University of Glasgow, Glasgow G12 8QQ, United Kingdom \\ ${ }^{2}$ Newman Laboratory of Nuclear Studies, Cornell University, Ithaca, New York 14853 \\ ${ }^{3}$ Department of Physics, Florida International University, Miami, Florida 33199 \\ ${ }^{4}$ Physics Department, The Ohio State University, Columbus, Ohio 43210 \\ ${ }^{5}$ Physics Department, University of Kentucky, Lexington, Kentucky 40506
}

(Received 13 July 2000; published 9 January 2001)

\begin{abstract}
We present results for the $B_{s}$ meson decay constant $f_{B_{s}}$ from simulations at three lattice spacings in the range $a^{-1}=1.1$ to $2.6 \mathrm{GeV}$ using NRQCD heavy quarks and clover light quarks in the quenched approximation. We study the scaling of this quantity and check the consistency between mesons decaying from rest and from a state with a nonzero spatial momentum. The cancellation of power law contributions that arise in the NRQCD formulation of heavy-light currents is discussed. On the coarsest lattice the $D_{s}$ meson decay constant $f_{D_{s}}$ is calculated. Our best values for the decay constants are given by $f_{B_{s}}=187(4)(4)(11)(2)(7)(6) \mathrm{MeV}$ and $f_{D_{s}}=223(6)(31)(38)(23)(9)\left({ }_{-1}^{+3}\right) \mathrm{MeV}$.
\end{abstract}

DOI: 10.1103/PhysRevD.63.034505

PACS number(s): 12.38.Gc, 13.20.Fc, 13.20.He

\section{INTRODUCTION}

The $B$ meson decay constant $f_{B}$ defined through the hadronic matrix element

$$
\left\langle 0\left|A_{\mu}\right| B, \vec{p}\right\rangle=p_{\mu} f_{B},
$$

is one of the phenomenologically relevant quantities whose determination has relied heavily on lattice calculations. Together with the bag parameter $B_{B}$, the decay constant is an important ingredient in analyses of $B^{0} \bar{B}^{0}$ mixing phenomena and studies of $C P$ violation in the neutral $B$ system. Several quenched lattice determinations of $f_{B}$ have been completed in recent years using different approaches to simulating the heavy $b$ quark on the lattice [1-8]. The first dynamical results are also starting to appear [9-11,6]. The most extensive quenched studies have been carried out using either the nonrelativistic QCD (NRQCD) formulation [12] or the heavy clover approach [13] to $b$ quarks. The results for $f_{B}$ and $f_{B_{s}}$ from these two methods are in good agreement with each other.

In this article we investigate once again the NRQCD approach to leptonic decays of heavy mesons. We wish to gain further insight into this method, which will strengthen confidence in our previous results and also allow us to assess its potential when going on to other important areas of heavy quark physics such as semileptonic decays. Our analysis is based on quenched results for the $B_{s}$ meson decay constant from simulations at three lattice spacings spanning $a^{-1}=1.1-2.6 \mathrm{GeV}$. We use NRQCD $b$ quarks, clover light quarks, and the Wilson plaquette gauge action. The simulation parameters are summarized in Table I. The $\beta=6.0 f_{B_{s}}$ results in Secs. III and IV are taken from Ref. [3]. The configurations at $\beta=5.7$ and $\beta=6.2$ plus the light propagators at

\footnotetext{
*Associated with the UKQCD Collaboration.

${ }^{\dagger}$ Present address: Spatial Technologies, Boulder, CO.
}

$\beta=6.2$ were provided by the UKQCD Collaboration. The $\beta=6.0$ data presented in Sec. VI also use UKQCD configurations and light propagators. Some preliminary results at earlier stages of this project have appeared in Refs. [18,19].

Our first goal was to check for the independence of our final $f_{B_{s}}$ value from the lattice spacing used. This is particularly important here, since we employ an effective theory NRQCD for heavy quarks which precludes extrapolations to zero lattice spacing $a \rightarrow 0$. There are many advantages to NRQCD, including being able to simulate quarks with masses much larger than the cutoff $\left(a M_{Q} \gg 1\right)$ without introducing large discretization errors and the efficiency with which heavy quark propagators can be computed numerically. However, the theory is not renormalizable and furthermore $a M_{Q}$ cannot become too small. In order for the approach to work, one must have a window in which the lattice spacing is large enough so that $a M_{Q}$ is of $O(1)$ or greater, but also small enough so that all discretization errors, including those coming from the glue and light quark sectors, are under control. Physical results must already be independent of the lattice spacing within acceptable and quantifiable systematic errors. The scaling studies presented in this article demonstrate that such a window does indeed exist between $a^{-1}=1.1-2.6 \mathrm{GeV}$ for simulations with NRQCD-clover quarks and Wilson glue. The systematic errors are $\sim 10 \%$ in the upper half of the window and increase to $\sim 20 \%$ towards the coarse end. By further improving the quark and gauge actions one should be able to improve the errors at the coarse end and even extend the window down below $1 \mathrm{GeV}$. Recently the JLQCD-Hiroshima Collaboration [4] studied scaling of $f_{B}$ and $f_{B_{s}}$ with a lattice action very similar to the one used in this article. Our results are in good agreement with theirs.

Another feature of the use of an effective theory, is that the matrix element of current operators in the full theory (continuum QCD) such as the LHS of Eq. (1) must be built up out of several current matrix elements evaluated in the effective theory: 
TABLE I. Simulation details for Secs. I-V. The errors on $a^{-1}$ include statistical errors and those due to the chiral extrapolation of $m_{\rho} . a M_{0}^{b}$ is the bare $b$-quark mass in lattice units determined from the $B$ (or $B_{s}$ ) meson. $\kappa_{q}$ gives the light quark mass for which results are presented here and $\kappa_{s}$ is the actual strange $\kappa$ for these $\beta$ values based on the $K$ meson. At $\beta=6.0$ time reversed configurations were also used and results were interpolated to $\kappa_{s}$.

\begin{tabular}{lccc}
\hline \hline & $\beta=5.7$ & $\beta=6.0$ & $\beta=6.2$ \\
\hline volume & $12^{3} \times 24$ & $16^{3} \times 48$ & $24^{3} \times 48$ \\
No. configs & 278 & $102 \times 2_{\text {trev }}$ & 144 \\
$c_{S W}$ & $1.567($ tad. imp. & 1.479 (tad. imp. & 1.61 (nonpert.) \\
$a^{-1}\left(m_{\rho}\right)(\mathrm{GeV})$ & $1.116(12)\left(_{-0}^{+56}\right)[14]$ & $1.92(7)[3]$ & $2.59\left({ }_{-10}^{+6}\right)\left({ }_{-0}^{+9}\right)[15-17]$ \\
$a M_{0}^{b}$ & $4.20(25)(5)\left(_{-24}^{+0}\right)[15]$ & $2.22(11)[3]$ & $1.64(5)\left({ }_{-5}^{+8}\right)\left(_{-7}^{+0}\right)[15]$ \\
$\kappa_{q}$ & 0.1400 & 0.13755 & 0.1346 \\
$\kappa_{s}$ & $0.1399(1)[16]$ & $0.13755(13)[3]$ & $0.13466(7)[16]$ \\
\hline \hline
\end{tabular}

$$
\left\langle A_{\mu}\right\rangle_{\mathrm{QCD}}=\sum_{j} C_{j}\left\langle J_{\mu}^{(j)}\right\rangle_{\mathrm{eff}} \cdot
$$

With NRQCD heavy quarks the currents are of the form

$$
J_{\mu}^{(j)}=\frac{1}{\left(M_{Q}\right)^{l_{j}}} O_{\mu}^{(j)},
$$

where the $O_{\mu}^{(j)}$ are operators of dimension $d_{j}=3+l_{j}$ (with $\left.l_{j} \geqslant 0\right)$. Quantum corrections to the currents and the mixing of higher dimension operators back onto lower dimension operators will induce "power law" terms, i.e., terms that go as $\alpha_{s} /\left(a M_{Q}\right)^{\delta l_{j}}$ (plus higher orders). Since the left-hand side (LHS) of Eq. (2) should have no power law contributions, any consistent framework for evaluating $\left\langle J_{\mu}^{(j)}\right\rangle_{\text {eff }}$ and $C_{j}$ must arrange for power law terms to cancel to the order one is working in. The second goal of this article was to investigate and quantify this cancellation at $O\left[\alpha_{s} /\left(a M_{Q}\right)\right]$. In our simulations we include all relevant current operators through dimension 4 . The matching coefficients $C_{j}$ have been calculated perturbatively through $O\left(\alpha_{s} / M_{Q}\right)$ and $O\left(a \alpha_{s}\right)$ [20]. Matrix elements of the dimension 4 current operators will contain in addition to $\Lambda_{\mathrm{QCD}} / M_{Q}$ physical contributions also power law terms of which the dominant $O\left[\alpha_{s} /\left(a M_{Q}\right)\right]$ terms are cancelled by our matching coefficients. Remaining uncancelled power law terms start at $O\left[\alpha_{s}^{2} /\left(a M_{Q}\right)\right]$ and their effects are taken into account in the systematic errors that we quote. Our analysis, based on the temporal component of the axial vector current $A_{0}$ demonstrates that although power law contributions are substantial there are no problems with cancelling them and there are no delicate fine tunings involved. Furthermore, we find that after cancellation of $O\left[\alpha_{s} /\left(a M_{Q}\right)\right]$ terms the contributions from $1 / M_{Q}$ current corrections are significantly reduced.

The third goal of the present study was to carry out other consistency tests in our lattice evaluation of $f_{B_{s}}$. The definition Eq. (1) of the decay constant tells us that one should be able to obtain the same $f_{B_{s}}$ from $B_{s}$ mesons at rest and from those with nonzero spatial momentum up to lattice artifacts. We have simulated leptonic decays of $B_{s}$ mesons with nonzero momenta to check this. Another project, which we will report on in a separate publication, is determining $f_{B_{s}}$ from the spatial component $A_{k}$, providing a further covariance test of calculations with NRQCD heavy quarks [21].

In the next section we introduce the lattice actions and current operators used in our simulations. Section III presents results for $f_{B_{s}}$ at the three lattice spacings and scaling plots. In Sec. IV we consider cancellation of power law contributions and also discuss the slope of $f_{P S} \sqrt{M_{P S}}$ versus $1 / M_{Q}$ as one leaves the static limit. On our coarsest lattice we are able to simulate NRQCD charm quarks and in Sec. V we present results for the $D_{s}$ meson decay constant $f_{D_{s}}$ at this one value for the lattice spacing. We describe $f_{B_{s}}$ extracted at nonzero momenta in Secs. VI and VII gives a brief summary of this article. In an appendix we provide a table of heavy-light current matching coefficients covering a wider range of heavy quark masses than in Ref. [20].

\section{THE LATTICE ACTION AND CURRENT OPERATORS}

We used the NRQCD heavy quark action density given by

$$
\begin{aligned}
\mathcal{L}= & \bar{\psi}_{t} \psi_{t}-\bar{\psi}_{t}\left(1-\frac{a \delta H}{2}\right)_{t}\left(1-\frac{a H_{0}}{2 n}\right)_{t}^{n} \\
& \times U_{4}^{\dagger}\left(1-\frac{a H_{0}}{2 n}\right)_{t-1}^{n}\left(1-\frac{a \delta H}{2}\right)_{t-1} \psi_{t-1},
\end{aligned}
$$

where $\psi$ denotes a two-component Pauli spinor, $H_{0}$ is the nonrelativistic kinetic energy operator

$$
H_{0}=-\frac{\Delta^{(2)}}{2 M_{0}}
$$

and $\delta H$ includes relativistic and finite-lattice-spacing corrections 
TABLE II. Bare heavy quark masses and $n$ values.

\begin{tabular}{|c|c|c|c|c|c|c|c|c|c|c|c|c|c|c|c|c|c|c|c|c|}
\hline & \multicolumn{20}{|c|}{$\beta=5.7$} \\
\hline$a M_{0}$ & 20.0 & 12.5 & 10.0 & 8.0 & 6.0 & 5.0 & 4.0 & 3.5 & 3.15 & 2.75 & 2.45 & 2.2 & 2.0 & 1.7 & 1.5 & 1.3 & 1.125 & 1.0 & 0.8 & 0.6 \\
\hline \multirow[t]{2}{*}{$n$} & 1 & 1 & 1 & 1 & 1 & 2 & 2 & 2 & 2 & 3 & 3 & 3 & 3 & 4 & 4 & 5 & 6 & 6 & 8 & 10 \\
\hline & \multicolumn{20}{|c|}{$\beta=6.0$} \\
\hline \multirow{3}{*}{$\begin{array}{l}a M_{0} \\
n\end{array}$} & & & 10.0 & & & 7.0 & & & 4.0 & & & 2.7 & & & 2.0 & & & 1.6 & & \\
\hline & & & 1 & & & 1 & & & 1 & & & 2 & & & 2 & & & 2 & & \\
\hline & \multicolumn{20}{|c|}{$\beta=6.2$} \\
\hline$a M_{0}$ & & & 4.5 & & & & & & 2.5 & & & & & & 1.44 & & & & & \\
\hline$n$ & & & 1 & & & & & & 3 & & & & & & 3 & & & & & \\
\hline
\end{tabular}

$$
\begin{aligned}
\delta H= & -c_{1} \frac{g}{2 M_{0}} \boldsymbol{\sigma} \cdot \mathbf{B}+c_{2} \frac{i g}{8\left(M_{0}\right)^{2}}(\boldsymbol{\nabla} \cdot \mathbf{E}-\mathbf{E} \cdot \boldsymbol{\nabla}) \\
& -c_{3} \frac{g}{8\left(M_{0}\right)^{2}} \boldsymbol{\sigma} \cdot(\boldsymbol{\nabla} \times \mathbf{E}-\mathbf{E} \times \boldsymbol{\nabla}) \\
& -c_{4} \frac{\left(\Delta^{(2)}\right)^{2}}{8\left(M_{0}\right)^{3}}+c_{5} \frac{a^{2} \Delta^{(4)}}{24 M_{0}}-c_{6} \frac{a\left(\Delta^{(2)}\right)^{2}}{16 n\left(M_{0}\right)^{2}} .
\end{aligned}
$$

In addition to all $1 / M^{2}$ terms we have also included the leading $1 / M^{3}$ relativistic correction as well as the discretization corrections appearing at the same order in the momentum expansion. For all three $\beta$ values the NRQCD action was tadpole improved using the plaquette definition of $u_{0}$ [22] and the $c_{i}$ were set equal to unity. Table II lists the bare heavy quark masses $a M_{0}$ at which simulations were carried out together with the corresponding values for the stability parameter $n$.

For the light quarks we used the tree-level tadpole improved clover action $\left(c_{S W}=u_{0}^{-3}\right)$ at $\beta=5.7$ and $\beta=6.0$. However at $\beta=6.2$ the nonperturbatively determined value $c_{S W}=1.61$ was employed [23]. The $\kappa$ values used in the simulations are listed in Table I. At $\beta=5.7$ and $\beta=6.2$ we employed a single $\kappa$ close to $\kappa_{s}$ fixed through the $K$ meson. At $\beta=6.0$ data was obtained at three light $\kappa$ 's 0.1369 , 0.1375 , and 0.13808 , and results were then interpolated to $\kappa_{s}$ [3]. In Table I we also list the lattice spacing determined from the $\rho$ mass and the bare dimensionless heavy quark mass $a M_{0}^{b}$ corresponding to the $b$ quark. The latter was determined from a combination of perturbation theory for mass renormalization and simulation results for binding energies. These procedures for fixing $a M_{0}^{b}$ have been explained in several previous publications $[3,15]$. The simulation parameters given in this paragraph apply to results presented in Secs. III, IV, and V. The $\beta=6.0$ simulations described in Sec. VI use different parameters (see Sec. VI and Table VII).

For the temporal component of the heavy-light axial vector current Eq. (2) becomes

$$
\left\langle A_{0}\right\rangle_{\mathrm{QCD}}=\sum_{j=0}^{2} C_{j}^{\left(A_{0}\right)}\left\langle J_{0}^{(j)}\right\rangle+O\left(1 / M_{Q}^{2}\right),
$$

with

$$
\begin{aligned}
& J_{0}^{(0)}=\bar{q} \gamma_{5} \gamma_{0} Q, \\
& J_{0}^{(1)}=\frac{-1}{2 M_{0}} \bar{q} \gamma_{5} \gamma_{0} \gamma \cdot \nabla Q, \\
& J_{0}^{(2)}=\frac{1}{2 M_{0}} \bar{q} \boldsymbol{\gamma} \cdot \stackrel{\nabla}{\nabla} \gamma_{5} \gamma_{0} Q
\end{aligned}
$$

The heavy quark 4-spinor $Q$ has the NRQCD 2-spinor $\psi$ as the upper two components, zero for the lower two components and obeys $\gamma_{0} Q=Q . J_{0}^{(0)}$ and $J_{0}^{(1)}$ contribute already at tree-level whereas $J_{0}^{(2)}$ appears only at one loop.

Higher dimension operators appear in lattice effective theories when one, for instance, needs to consider relativistic corrections. They also occur whenever lattice operators are improved. Improvements to current operators take on the form

$$
J_{\mu}^{(j) \mathrm{disc}}=a^{l_{j}} O_{\mu}^{(j)}
$$

where again, as in Eq. (3), the $O_{\mu}^{(j)}$ are operators of dimension $d_{j}=3+l_{j}$. In the present case of NRQCD-clover currents, one has the identical set of possible dimension 4 operators $O_{\mu}^{(j)}$ in Eqs. (3) and (9). Hence a one-loop mixing and matching calculation to full continuum QCD involving the above $J_{\mu}^{(j)}$ will not only tell us how the $1 / M_{Q}$ currents contribute but will also identify the discretization corrections that come in at one loop. In other words a consistent matching through $O\left(\alpha_{s} / M_{Q}\right)$ should automatically be consistent through $O\left(a \alpha_{s}\right)$. In Ref. [20] it was found that for each $\mu$ only one of the $J_{\mu}^{(j) \text { disc }}$ was relevant at one loop. Matching to continuum QCD could be achieved by improving $J_{\mu}^{(0)}$ in the following way:

$$
J_{\mu}^{(0)} \rightarrow J_{\mu}^{(0)}+\alpha_{s} \zeta_{A_{\mu}} J_{\mu}^{(\mathrm{disc})}
$$

with

$$
J_{\mu}^{(\mathrm{disc})}=-a \bar{q} \boldsymbol{\gamma} \cdot \stackrel{\nabla}{\nabla} \gamma_{0} \gamma_{5} \gamma_{\mu} Q=2 a M_{0} J_{\mu}^{(2)},
$$


TABLE III. Estimates of the statistical and main systematic errors, in percent, in our values for $f_{B_{s}}$.

\begin{tabular}{lrrr}
\hline \hline Source & $\beta=5.7$ & $\beta=6.0$ & $\beta=6.2$ \\
\hline statistical & 3 & 3 & 2 \\
disc. $O\left[\left(a \Lambda_{\mathrm{QCD}}\right)^{2}\right]$ & 13 & 4 & 2 \\
disc. $O\left[\alpha_{s}\left(a \Lambda_{\mathrm{QCD}}\right)\right]$ & 5 & 2 & 6 \\
pert. $O\left(\alpha_{s}^{2}\right), O\left[\alpha_{s}^{2} /(a M)\right]$ & 11 & 7 & 1 \\
NRQCD $O\left[\left(\Lambda_{\mathrm{QCD}} / M\right)^{2}\right], O\left(\alpha_{s} \Lambda_{\mathrm{QCD}} / M\right)$ & 1 & 1 & 3 \\
$\kappa_{s}$ & +4 & +4 & 8 \\
$a^{-1}\left(m_{\rho}\right)$ & $\left(\begin{array}{c}+5 \\
-1\end{array}\right)$ & 4 & 8 \\
Total & & & \\
\hline \hline
\end{tabular}

and $\zeta_{A_{0}}=1.03$. The effect of $J_{\mu}^{(\text {disc })}$ can be taken into account by expressing it in terms of $J_{\mu}^{(2)}$ and absorbing $\alpha_{s} 2 a M_{0} \zeta_{A_{\mu}}$ into the coefficient $C_{2}^{\left(A_{\mu}\right)}$.

At $\beta=5.7$ and $\beta=6.01 / M_{Q}^{2}$ current corrections that appear at tree-level have also been studied. For $A_{0}$ these are

$$
\begin{aligned}
& J_{0}^{(3)}=\frac{1}{8 M_{0}^{2}} \bar{q} \gamma_{5} \gamma_{0} \mathbf{D}^{2} Q, \\
& J_{0}^{(4)}=\frac{g}{8 M_{0}^{2}} \bar{q} \gamma_{5} \gamma_{0} \mathbf{\Sigma} \cdot \mathbf{B} Q, \\
& J_{0}^{(5)}=\frac{-i g}{4 M_{0}^{2}} \bar{q} \gamma_{5} \gamma_{0} \boldsymbol{\alpha} \cdot \mathbf{E} Q,
\end{aligned}
$$

where $\boldsymbol{\alpha} \equiv \gamma_{0} \boldsymbol{\gamma}$ and $\boldsymbol{\Sigma}=\operatorname{diag}(\boldsymbol{\sigma}, \boldsymbol{\sigma})$. We will comment on matrix elements of these currents and their $M_{Q}$ dependence later in Sec. V. However, since the $O\left(\alpha_{s} / M_{Q}^{2}\right)$ full matching calculation has not been carried out yet $O\left[\alpha_{s} /\left(a M_{Q}\right)^{2}\right]$ and $O\left[\alpha_{s}\left(\Lambda_{\mathrm{QCD}} / M_{Q}\right) /\left(a M_{Q}\right)\right]$ power law contaminations contained in these matrix elements will not be cancelled and we prefer not to include them in our scaling analysis of $f_{B_{s}}$. In Ref. [3] tree-level contributions from Eq. (12) were included. The total effect was a $\sim 3 \%$ decrease in $f_{B}$, well within the systematic errors quoted there for uncancelled power law terms. In the $\beta=6.0$ results given in the present article, however, we have removed the $1 / M_{Q}^{2}$ tree-level current effects.

Based on the lattice actions employed, the improvement of $J_{\mu}^{(0)}$ and the consistent matching to continuum full QCD through $O\left(\alpha_{s} / M_{Q}\right)$ and $O\left(a \alpha_{s}\right)$ in the currents, one can now list the expected remaining dominant systematic errors in our evaluation of $\left\langle A_{\mu}\right\rangle_{\mathrm{QCD}}$. These are collected in Table III and their size, in percent, estimated for each $\beta$. In making these estimates we use $\Lambda_{\mathrm{QCD}} \sim 400 \mathrm{MeV}, \alpha_{s}(\beta=5.7)$ $\sim 0.33, \alpha_{s}(\beta=6.0) \sim 0.24, \alpha_{s}(\beta=6.2) \sim 0.20$ and approximate $a M_{Q}$ with $a M_{0}^{b}$ of Table I. The $\alpha_{s}$ values are close to $\alpha_{P}\left(q^{*}=1 / a\right)$, where $\alpha_{P}$ is the coupling introduced in Ref. [24] closely related to $\alpha_{V}$ which is based on the heavy quark potential [25,22]. Discretization errors come in at $O\left[\left(a \Lambda_{\mathrm{QCD}}\right)^{2}\right]$ from the currents and at $\beta=5.7$ and $\beta=6.0$ also at $O\left(\alpha_{s} a \Lambda_{\mathrm{QCD}}\right)$ from the light quark action. These latter errors arise from using a tadpole-improved $c_{S W}$ rather than a nonperturbative $c_{S W}$ (or a $c_{S W}$ value fully corrected at oneloop) and are therefore proportional to the difference of these values for $c_{S W}$. Such differences and, hence, also the $O\left(\alpha_{s} a \Lambda_{\mathrm{QCD}}\right)$ errors are small at $\beta=6.0$. Relativistic errors are at $O\left[\left(\Lambda_{\mathrm{QCD}} / M_{Q}\right)^{2}\right]$ from the currents. There are also $O\left(\alpha_{s} \Lambda_{\mathrm{QCD}} / M_{Q}\right)$ errors from using the tadpole-improved coefficient for the $\sigma \cdot B$ term in the NRQCD action instead of the full $O\left(\alpha_{s}\right)$ coefficient matched to continuum QCD. This error is proportional to the $1 / M_{Q}$ slope induced in $f_{B}$ by the $\sigma \cdot B$ term. The investigations in Ref. [9] found this slope to be small, approximately $0.25 \times \Lambda_{\mathrm{QCD}} / M_{Q}$. We fold in perturbative errors separately for $O\left(\alpha_{s}^{2}\right)$ and $O\left[\alpha_{s}^{2} /\left(a M_{Q}\right)\right]$ since these have different $\beta$ dependence and also to indicate that perturbation theory will eventually break down for small $a M_{Q}$, i.e., for large enough $\beta$. We note that in the absence of tree-level $O\left[1 /\left(M_{Q}^{2}\right)\right]$ current matrix elements, there are no $O\left[\alpha_{s} /\left(a M_{Q}\right)^{2}\right]$ errors in our calculations. As long as the same action is used in both the simulations and the perturbative matchings, all $O\left[\alpha_{s} /\left(a M_{Q}\right)^{j}\right]$ terms cancel between matrix elements and matching coefficients. Table III also lists other systematic errors (uncertainty in $\kappa_{s}$ and fixing of $a^{-1}$ from the $\rho$ mass) and the statistical errors in the $f_{B_{s}}$ determinations. Not listed are uncertainties due to quenching. Recent studies indicate that these last corrections can be substantial, at the 10-25\% level [9-11].

\section{SIMULATION RESULTS AND SCALING OF $\boldsymbol{f}_{B_{s}}$}

In this section we describe $f_{B}$ calculated from $A_{0}$ and investigate scaling of this quantity. We start with simulation results for current matrix elements in lattice units before renormalization. Table IV summarizes our results at $\beta=5.7$ and $\beta=6.2$ for

$$
a^{3 / 2} f^{(j)} \sqrt{M_{P S}}=\frac{a^{3 / 2}}{\sqrt{M_{P S}}}\left\langle 0\left|J_{0}^{(j)}\right| P S\right\rangle .
$$

Data are presented for several heavy quark masses with the light quark mass fixed to $\kappa_{q}$ of Table I. The corresponding simulation data for $\beta=6.0$ can be found in Ref. [3]. The matrix elements were evaluated in the meson rest frame. $\left\langle J_{0}^{(2)}\right\rangle$ is identical to $\left\langle J_{0}^{(1)}\right\rangle$ in this frame and hence is not 
TABLE IV. Matrix elements of individual current contributions in lattice units. $f^{(j)}$ is defined in Eq. (13). The $\beta=6.2$ matrix elements have different normalization from those at other $\beta$ 's (see text).

\begin{tabular}{|c|c|c|c|c|c|}
\hline$a M_{0}$ & $a^{3 / 2} f^{(0)} \sqrt{M}$ & $a^{3 / 2} f^{(1)} \sqrt{M}$ & $a^{3 / 2} f^{(3)} \sqrt{M}$ & $a^{3 / 2} f^{(4)} \sqrt{M}$ & $a^{3 / 2} f^{(5)} \sqrt{M}$ \\
\hline 0.600 & $0.455(7)$ & $-0.188(4)$ & $-0.229(4)$ & $0.1148(20)$ & $-0.1463(24)$ \\
\hline 0.800 & $0.445(7)$ & $-0.1448(27)$ & $-0.1145(21)$ & $0.0583(10)$ & $-0.0763(12)$ \\
\hline 1.000 & $0.445(8)$ & $-0.1204(24)$ & $-0.0678(13)$ & $0.0348(6)$ & $-0.0470(8)$ \\
\hline 1.125 & $0.449(8)$ & $-0.1105(23)$ & $-0.0535(11)$ & $0.0267(5)$ & $-0.0369(7)$ \\
\hline 1.300 & $0.455(9)$ & $-0.0992(22)$ & $-0.0395(9)$ & $0.0193(4)$ & $-0.0274(6)$ \\
\hline 1.500 & $0.462(9)$ & $-0.0893(21)$ & $-0.0297(7)$ & $0.0141(3)$ & $-0.0205(4)$ \\
\hline 1.700 & $0.471(10)$ & $-0.0820(20)$ & $-0.0238(6)$ & $0.01072(25)$ & $-0.0161(4)$ \\
\hline 2.000 & $0.484(11)$ & $-0.0731(19)$ & $-0.0179(5)$ & $0.00754(19)$ & $-0.01170(27)$ \\
\hline 2.200 & $0.492(11)$ & $-0.0685(18)$ & $-0.0153(4)$ & $0.00616(17)$ & $-0.00975(23)$ \\
\hline 2.450 & $0.503(12)$ & $-0.0636(18)$ & $-0.0130(4)$ & $0.00490(14)$ & $-0.00795(19)$ \\
\hline 2.750 & $0.515(13)$ & $-0.0587(17)$ & $-0.0108(3)$ & $0.00385(12)$ & $-0.00639(15)$ \\
\hline 3.150 & $0.528(14)$ & $-0.0533(16)$ & $-0.00874(25)$ & $0.00289(10)$ & $-0.00493(12)$ \\
\hline 3.500 & $0.541(15)$ & $-0.0495(16)$ & $-0.00748(23)$ & $0.00233(9)$ & $-0.00405(10)$ \\
\hline 4.000 & $0.556(17)$ & $-0.0450(16)$ & $-0.00614(20)$ & $0.00177(7)$ & $-0.00315(8)$ \\
\hline 5.000 & $0.583(21)$ & $-0.0381(15)$ & $-0.00440(16)$ & $0.00112(5)$ & $-0.00207(6)$ \\
\hline 6.000 & $0.602(25)$ & $-0.0331(15)$ & $-0.00331(13)$ & $0.00077(5)$ & $-0.00146(4)$ \\
\hline 8.000 & $0.64(3)$ & $-0.0263(15)$ & $-0.00213(10)$ & $0.00043(3)$ & $-0.00085(3)$ \\
\hline 10.000 & $0.66(4)$ & $-0.0218(14)$ & $-0.00149(9)$ & $0.000272(25)$ & $-0.000555(25)$ \\
\hline 12.500 & $0.68(6)$ & $-0.0180(14)$ & $-0.00103(7)$ & $0.000171(19)$ & $-0.000362(20)$ \\
\hline 20.000 & $0.73(9)$ & $-0.0119(13)$ & $-0.00045(4)$ & $0.000066(11)$ & $-0.000144(11)$ \\
\hline \multicolumn{6}{|c|}{$\beta=6.2$} \\
\hline 1.440 & $0.143(2)$ & $-0.0195(3)$ & & & \\
\hline 2.500 & $0.147(2)$ & $-0.0125(2)$ & & & \\
\hline 4.500 & $0.152(1)$ & $-0.0076(1)$ & & & \\
\hline
\end{tabular}

listed separately in the table. For $\beta=5.7$ we also list results for the higher order currents of Eq. (12).

Results in physical units $\left(\mathrm{GeV}^{3 / 2}\right)$ for

$$
f_{P S} \sqrt{M_{P S}}=\sum_{j=0}^{2} C_{j}^{\left(A_{0}\right)} f^{(j)} \sqrt{M_{P S}}
$$

are summarized in Table $\mathrm{V}$, at tree-level $\left(C_{0}^{\left(A_{0}\right)}=C_{1}^{\left(A_{0}\right)}=1\right.$, $\left.C_{2}^{\left(A_{0}\right)}=0\right)$ and with one-loop matching for three different scales to fix $\alpha_{s}=\alpha_{P}\left(q^{*}\right)$. At $\beta=5.7$ and $\beta=6.0$ we can directly take over the matching coefficients from the Appendix, which assume tadpole improvement in both the NRQCD and clover actions. At $\beta=6.2$ where the nonperturbative $c_{S W}$ is used, some slight modifications are necessary. We first remove the tadpole improvement term coming from the light quark wave function renormalization (denoted $C_{q}^{T I}$ in Ref. [20]) from the matching coefficients. We then use $\kappa$ rather than $\tilde{\kappa}=\kappa \times u_{0}$ to rescale the light quark field in the currents. As a result the entries in Table IV and the tree-level results in Table $\mathrm{V}$ are enhanced for $\beta=6.2$ by approximately a factor of $u_{0}^{-1 / 2}$. This enhancement is compensated for to a large extent upon going to renormalized matrix elements (the latter three columns in Table V) due to larger perturbative matching coefficients. Alternatively, we could have used another light quark action at $\beta=6.2$ which has the first derivative terms divided by $u_{0}$ and a clover term with coefficient $C_{S W}^{\text {nonpert. }} / u_{0}$. This would have allowed us to use tadpoleimproved perturbation theory even for the light quark propagator, similar to what was done at the other $\beta$ values. We tested this option and found that the final answer for the decay constant changed by $4-5 \%$. This difference is, as expected, of the same order as other $O\left(\alpha_{s}^{2}\right)$ corrections not included in the present analysis.

The scale $q^{*}$ has not been calculated yet for the matching coefficients with NRQCD heavy and clover light quarks. $q^{*}$ is known for static heavy quarks combined with massless Wilson light quarks [26] and also for light-light currents [27] again using massless Wilson fermions. These authors find $q^{*}=2.18 / a$ and $q^{*}=2.3 / a$, respectively, suggesting very mild dependence of $q^{*}$ on $a M_{Q}$. Results also exist for clover light-light currents [28] showing some reduction in $q^{*}$ relative to the Wilson results for these currents. In the absence of an explicit calculation for the action and currents employed in the present simulations, we use the $q^{*}=2 / a$ numbers to quote central values and arrange for perturbative errors to cover the spread in results due to uncertainty in $q^{*}$. Table VI shows our final numbers for $f_{B_{s}}$ interpolated to the physical heavy quark mass $M_{0}^{b}$ for the three lattice spacings. 
TABLE V. Decay matrix elements $f \sqrt{M}$ in $\mathrm{GeV}^{3 / 2}$. The first column gives tree-level results, the remaining columns include renormalization constants using $\alpha_{P}$ at three values of $q^{*}$. Only statistical errors are shown.

\begin{tabular}{|c|c|c|c|c|}
\hline$a M_{0}$ & tree-level & $\begin{array}{r}q^{*}=1 / a \\
\beta=\end{array}$ & $q^{*}=2 / a$ & $q^{*}=\pi / a$ \\
\hline 0.600 & $0.315(6)$ & $0.263(5)$ & $0.280(5)$ & $0.286(5)$ \\
\hline 0.800 & $0.353(6)$ & $0.280(5)$ & $0.304(5)$ & $0.312(5)$ \\
\hline 1.000 & $0.382(7)$ & $0.302(5)$ & $0.328(6)$ & $0.337(6)$ \\
\hline 1.125 & $0.399(7)$ & $0.314(6)$ & $0.341(6)$ & $0.351(6)$ \\
\hline 1.300 & $0.419(8)$ & $0.329(6)$ & $0.358(7)$ & $0.369(7)$ \\
\hline 1.500 & $0.440(9)$ & $0.345(7)$ & $0.375(8)$ & $0.386(8)$ \\
\hline 1.700 & $0.459(10)$ & $0.364(8)$ & $0.394(9)$ & $0.406(9)$ \\
\hline 2.000 & $0.484(11)$ & $0.378(9)$ & $0.412(10)$ & $0.424(10)$ \\
\hline 2.200 & $0.500(11)$ & $0.385(9)$ & $0.422(10)$ & $0.435(10)$ \\
\hline 2.450 & $0.518(12)$ & $0.396(10)$ & $0.435(10)$ & $0.449(11)$ \\
\hline 2.750 & $0.538(14)$ & $0.408(10)$ & $0.450(11)$ & $0.465(12)$ \\
\hline 3.150 & $0.560(15)$ & $0.421(12)$ & $0.466(13)$ & $0.482(13)$ \\
\hline 3.500 & $0.579(17)$ & $0.432(13)$ & $0.479(14)$ & $0.496(14)$ \\
\hline 4.000 & $0.603(19)$ & $0.446(14)$ & $0.496(16)$ & $0.514(16)$ \\
\hline 5.000 & $0.642(24)$ & $0.471(18)$ & $0.526(19)$ & $0.546(20)$ \\
\hline 6.000 & $0.671(28)$ & $0.488(21)$ & $0.547(23)$ & $0.568(24)$ \\
\hline 8.000 & $0.72(4)$ & $0.519(28)$ & $0.58(3)$ & $0.61(3)$ \\
\hline 10.000 & $0.75(5)$ & $0.55(4)$ & $0.61(4)$ & $0.64(4)$ \\
\hline 12.500 & $0.79(7)$ & $0.57(5)$ & $0.64(6)$ & $0.67(6)$ \\
\hline \multirow[t]{2}{*}{20.000} & $0.84(10)$ & $0.63(9)$ & $0.70(10)$ & $0.72(10)$ \\
\hline & \multicolumn{4}{|c|}{$\beta=6.0$} \\
\hline 1.600 & $0.442(13)$ & $0.398(11)$ & $0.410(11)$ & $0.414(11)$ \\
\hline 2.000 & $0.466(13)$ & $0.406(13)$ & $0.421(13)$ & $0.427(13)$ \\
\hline 2.700 & $0.495(19)$ & $0.421(16)$ & $0.440(16)$ & $0.448(16)$ \\
\hline 4.000 & $0.521(19)$ & $0.442(16)$ & $0.465(16)$ & $0.473(16)$ \\
\hline 7.000 & $0.588(16)$ & $0.479(13)$ & $0.508(13)$ & $0.518(13)$ \\
\hline \multirow[t]{2}{*}{10.000} & $0.615(16)$ & $0.498(13)$ & $0.528(13)$ & $0.540(13)$ \\
\hline & \multicolumn{4}{|c|}{$\beta=6.2$} \\
\hline 1.440 & $0.515(8)$ & $0.404(4)$ & $0.429(8)$ & $0.442(8)$ \\
\hline 2.500 & $0.561(8)$ & $0.465(6)$ & $0.485(6)$ & $0.495(6)$ \\
\hline 4.500 & $0.602(4)$ & $0.505(5)$ & $0.527(5)$ & $0.536(5)$ \\
\hline
\end{tabular}

The Particle Data Group's $B_{s}^{0}$ mass, $5.369 \mathrm{GeV}$, was used to convert from $f_{P S} \sqrt{M_{P S}}$ to $f_{B_{s}}$. The errors follow those listed in Table III and correspond from left to right to statistical (plus interpolation in $M_{0}$ ), discretization, perturbative, relativistic, $\kappa_{s}$ determination and errors in $a^{-1}\left(m_{\rho}\right)$.

After adding all errors in quadrature, we plot $f_{B_{s}}$ versus $a$ in Fig. 1 and compare with results from Ref. [4]. One finds good scaling within errors and excellent agreement between the two collaborations. These results also agree well with quenched $f_{B_{s}}$ determinations using other heavy fermion formulations on the lattice [1].

Table VI and Fig. 1 represent one of the main results of this article. Working around $a^{-1}$ of $\sim 2 \mathrm{GeV}$ with NRQCDclover quarks and Wilson glue and matching through $O\left(\alpha_{s} / M_{Q}\right)$ and $O\left(a \alpha_{s}\right)$, one can obtain reliable quenched
TABLE VI. Quenched $f_{B_{s}}$ with errors listed separately and added in quadrature to the right. The errors denote from left to right statistical (plus interpolations in $M_{0}$ ), discretization, perturbative, relativistic, fixing $\kappa_{s}$ and $a^{-1}$.

\begin{tabular}{llll}
\hline \hline$\beta$ & \multicolumn{3}{c}{$f_{B_{s}}$ in $\mathrm{MeV}$} \\
\hline 5.7 & $217(7)(30)(24)(2)(9)\left({ }_{-3}^{+12}\right)$ & $\rightarrow$ & $217\left({ }_{-40}^{+42}\right)$ \\
6.0 & $184(6)(8)(13)(2)(7)(7)$ & $\rightarrow$ & $184(19)$ \\
6.2 & $187(4)(4)(11)(2)(7)(6)$ & $\rightarrow$ & $187(16)$ \\
\hline \hline
\end{tabular}

numbers for $B$ meson decay constants with $\sim 10 \%$ accuracy. To do better at these lattice spacings one would need to go to higher orders in the matching procedure. At our coarsest lattice spacing the systematic uncertainties are larger. Going to more highly improved actions would help here. Nevertheless, the $\beta=5.7$ results are consistent with our best numbers. Simulations with $a^{-1} \sim 1 \mathrm{GeV}$ can already give a good indication of continuum physics.

Since we cannot extrapolate to $a \rightarrow 0$, in order to quote our best estimate for quenched $f_{B_{s}}$ there are several choices. One approach would be to average over the $\beta=6.0$ and $\beta$ $=6.2$ results. Alternatively one could, after having verified scaling behavior, pick the point with the smallest statistical and systematic errors. Based on Table III this corresponds to the $\beta=6.2$ result. We adopt the second method here and present, as our final best value for the $B_{s}$ meson decay constant in the quenched approximation

$$
f_{B_{s}}=187(4)(4)(11)(2)(7)(6) \mathrm{MeV},
$$

with errors as described in Table VI.

If the value of $f_{B_{s}}$ at the physical $b$-quark mass were our only goal then we would be done at this point. However, if further details are of interest, such as "the true size of contributions from $1 / M_{Q}$ current corrections to $f_{B}$ " or "the

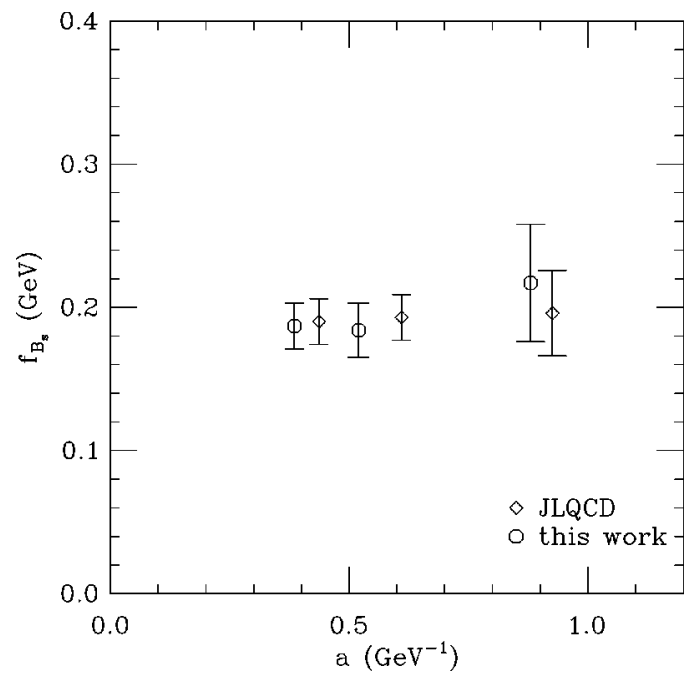

FIG. 1. $f_{B_{s}}$ versus the lattice spacing and comparison with Ref. [4]. Systematic and statistical errors have been added in quadrature. 
slope of $f_{P S} \sqrt{M_{P S}}$ versus $1 / M_{Q}$," then one needs to scrutinize more carefully the contributions from individual current matrix elements $\left\langle J_{0}^{(j)}\right\rangle$ and matching coefficients $C_{j}$ to the RHS of Eq. (7). We turn to these issues in the next section.

\section{POWER LAW TERMS AND $\Lambda_{\mathrm{QCD}} / M_{Q}$ CORRECTIONS}

In this section we investigate further the properties of matrix elements of the $1 / M_{Q}$ current corrections. We focus on the current $J_{0}^{(1)}=\left(-1 / 2 M_{0}\right) \bar{q} \gamma_{5} \gamma_{0} \boldsymbol{\gamma} \cdot \boldsymbol{\nabla} Q$. This operator is introduced in order to take into account $p / M_{Q}$ effects which we know are present in the full theory, i.e., in the LHS of Eq. (7). Here " $p$ " is the momentum of the quarks inside the meson and should be of $O\left(\Lambda_{\mathrm{QCD}}\right)$. Once introduced into the effective theory, however, this current will mix with other currents in the theory. Its short distance effect will be to renormalize the zeroth order current and one expects the matrix element $\left\langle J_{0}^{(1)}\right\rangle$ to develop a component proportional to $\left\langle J_{0}^{(0)}\right\rangle$. On dimensional grounds the proportionality coefficient will be of the form const/ $\left(a M_{Q}\right)$ and one has

$$
\left\langle J_{0}^{(1)}\right\rangle=\frac{C_{10}}{a M_{Q}}\left\langle J_{0}^{(0)}\right\rangle+\frac{\Lambda_{\mathrm{QCD}}}{M_{Q}} \text { term }+ \text { higher orders. }
$$

The first term on the RHS of Eq. (16) is an example of a power law contribution to a matrix element. They are unavoidable in a quantum effective field theory where one has mixing between operators of different dimensions. However, the matching procedures between the full and the effective theories should remove these artifacts. Since we do the matching perturbatively, power law contributions can only be subtracted to a given order in $\alpha_{s}$. As mentioned several times already, in our present calculations we cancel terms through $O\left[\alpha_{s} /\left(a M_{Q}\right)\right]$. To see precisely how this cancellation comes about we need to dissect the matching coefficient $C_{j=0}$. Using the notation of Ref. [20] one can rewrite Eq. (7) as

$$
\left\langle A_{0}\right\rangle_{\mathrm{QCD}}=\left(1+\alpha_{s} \rho_{0}\right)\left\langle J_{0}^{(0)}\right\rangle+\left(1+\alpha_{s} \rho_{1}\right)\left\langle J_{0}^{(1)}\right\rangle+\alpha_{s} \rho_{2}\left\langle J_{0}^{(2)}\right\rangle,
$$

with

$$
\rho_{0}=B_{0}-\frac{1}{2}\left(C_{q}+C_{Q}\right)-\zeta_{00}-\zeta_{10} \equiv \tilde{\rho}_{0}-\zeta_{10}
$$

$\rho_{0}$ is the one-loop contribution to $C_{0}$ and consists of parts coming from continuum perturbation theory $\left(B_{0}\right)$, from light and heavy quark wave function renormalizations on the lattice $\left(C_{q}\right.$ and $\left.C_{Q}\right)$ and from mixing between the currents $J_{0}^{(j)}$ $\left(\zeta_{00}\right.$ and $\left.\zeta_{10}\right) . \zeta_{00}$ is the feedback of $J_{0}^{(0)}$ onto itself and $\zeta_{10}$ incapsulates the projection of $J_{0}^{(1)}$ back onto $J_{0}^{(0)}$, precisely what one is looking for in the first term of Eq. (16). Picking out the relevant terms, one can write

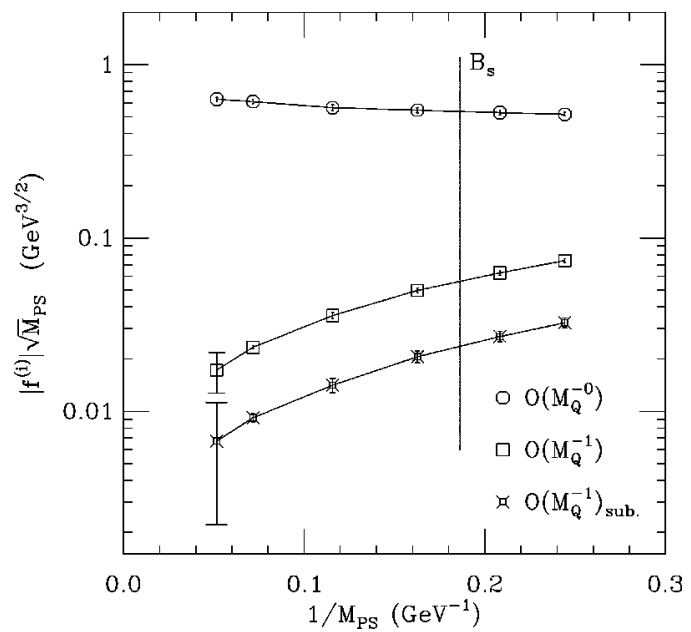

FIG. 2. Matrix elements $f^{(0)} \sqrt{M},\left|f^{(1)}\right| \sqrt{M}$, and $\left|f_{\text {sub }}^{(1)}\right| \sqrt{M}$ versus $1 / M_{P S}$ at $\beta=6.0 . q^{*}=2 / a$ was used to obtain $\left|f_{\text {sub }}^{(1)}\right| \sqrt{M}$.

$$
\begin{aligned}
\left(1+\alpha_{s} \rho_{0}\right)\left\langle J_{0}^{(0)}\right\rangle+\left\langle J_{0}^{(1)}\right\rangle & \\
= & \left(1+\alpha_{s} \tilde{\rho}_{0}\right)\left\langle J_{0}^{(0)}\right\rangle+\left[\frac{C_{10}}{a M_{Q}}-\alpha_{s} \zeta_{10}\right]\left\langle J_{0}^{(0)}\right\rangle \\
& +\frac{\Lambda_{\mathrm{QCD}}}{M_{Q}} \operatorname{term}+\cdots
\end{aligned}
$$

The power law contribution $\left[C_{10} / a M_{Q}-\alpha_{s} \zeta_{10}\right]\left\langle J_{0}^{(0)}\right\rangle$ has now been reduced from an $O\left[\alpha_{s} /\left(a M_{Q}\right)\right]$ to an $O\left[\alpha_{s}^{2} /\left(a M_{Q}\right)\right]$ effect through the $\alpha_{s} \zeta_{10}$ matching term. It is convenient to define a subtracted $J_{0}^{(1)}$,

$$
\left\langle J_{0}^{(1)}\right\rangle_{\mathrm{sub}}=\left\langle J_{0}^{(1)}\right\rangle-\alpha_{s} \zeta_{10}\left\langle J_{0}^{(0)}\right\rangle
$$

which is equal to the last two terms in Eq. (19) up to higher order corrections. It is $\left\langle J_{0}^{(1)}\right\rangle_{\text {sub }}$ rather than $\left\langle J_{0}^{(1)}\right\rangle$ that should be used to assess the importance of $1 / M_{Q}$ current corrections to $f_{B_{s}}$. In Fig. 2 we plot the absolute values of $f^{(0)} \sqrt{M}$, $f^{(1)} \sqrt{M}$ and $f_{\text {sub }}^{(1)} \sqrt{M}$ (related to $\left\langle J_{0}^{(0)}\right\rangle,\left\langle J_{0}^{(1)}\right\rangle$, and $\left\langle J_{0}^{(1)}\right\rangle_{\text {sub }}$ in the usual way) versus $1 / M_{P S}$ at $\beta=6.0$. One sees that $\left|f_{\text {sub }}^{(1)}\right| \sqrt{M}$ is considerably smaller than $\left|f^{(1)}\right| \sqrt{M}$ and amounts to only $\sim 4-\sim 5 \%$ of the lowest order $f^{(0)} \sqrt{M}$ term. This is of the order of systematic errors such as $O\left(\alpha_{s}^{2}\right)$ errors remaining in our calculation, so we cannot give a more precise estimate of the true $\Lambda_{\mathrm{QCD}} / M_{Q}$ contributions coming from $1 / M_{Q}$ currents. Similar results are obtained at the other lattice spacings. A corresponding plot similar to Fig. 2 for $\beta$ $=5.7$ will be discussed in the next section. At all three $\beta$ values the power law contributions make up $50 \%-80 \%$ of $\left\langle J_{0}^{(1)}\right\rangle$, depending on $q^{*}$, and once they are subtracted away one is left with only a small remainder. We note that the subtraction in Eq. (20) took place automatically in the $f_{B_{s}}$ calculations of the previous section. The fact that $f_{\text {sub }}^{(1)} \sqrt{M}$ turned out to be small did not pose any problems for the power law cancellation itself at $O\left(\alpha_{s} /\left(a M_{Q}\right)\right)$. 


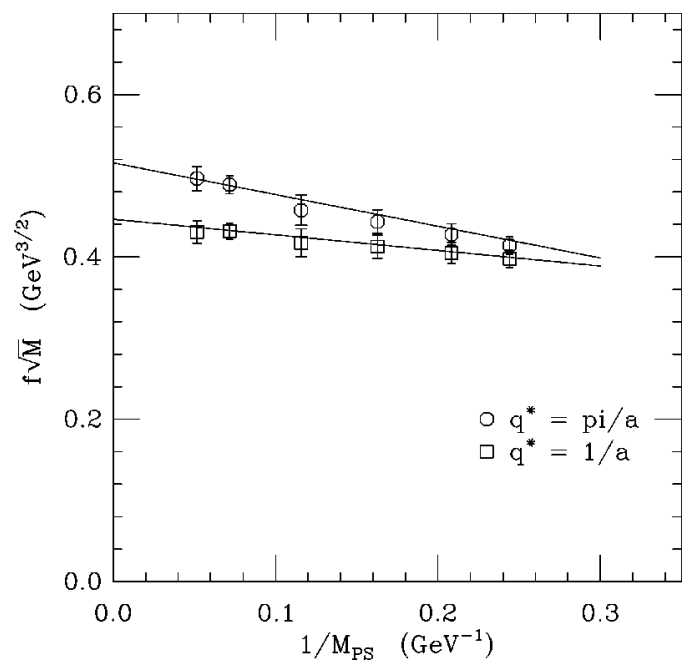

FIG. 3. $f_{P S} \sqrt{M_{P S}}$ versus $1 / M_{P S}$ at $\beta=6.0$ for $q^{*}=\pi / a$ and $q^{*}=1 / a$. The lines are linear fits to the data. Only statistical errors are shown.

Another quantity where having information on $\Lambda_{\mathrm{QCD}} / M_{Q}$ corrections is important, is the slope of $f_{P S} \sqrt{M_{P S}}$ versus $1 / M_{Q}$. Writing

$$
f_{P S} \sqrt{M_{P S}}=\left(f_{P S} \sqrt{M_{P S}}\right)_{\text {stat }}\left[1+\frac{\Lambda_{s l}}{M_{Q}}+O\left(1 / M_{Q}^{2}\right)\right],
$$

one is interested in the slope $\Lambda_{s l}$. The bulk of the effect will come from $1 / M_{Q}$ terms in the NRQCD action which are responsible for the $M_{O}$ dependence of the zeroth order current matrix element $\left\langle J_{0}^{(0)}\right\rangle$, and from the one-loop correction $\tilde{\rho}_{0}$ to this matrix element. We have just seen that contributions from $1 / M_{Q}$ current corrections are small. Nevertheless, when simulation data are fit to the form (21) to extract $\Lambda_{s l}$ one might worry about contaminations due to uncancelled power law terms of $O\left(\alpha_{s}^{2} /\left(a M_{Q}\right)\right)$. If $\Lambda_{s l}$ is the result of a fit then one can estimate a percentage error of $\alpha_{s}^{2} /\left(a \Lambda_{s l}\right)$ in this slope. Putting in some numbers this leads to $10-13 / \Lambda_{s l}[\mathrm{GeV}] \%$ uncertainties depending on $\beta$, where $\Lambda_{s l}$ must be inserted in GeV's. If the slope is less than $\Lambda_{\mathrm{QCD}}(0.4$ $\mathrm{GeV}$ ) these errors can become quite large.

There is, however, another reason why it is difficult to extract an accurate $\Lambda_{s l}$ from our simulations. In order to calculate the slope we need to look at data at masses larger than the $b$ quark mass. The one-loop terms in the matching coefficients increase as one goes towards the static limit and as a consequence results for $f_{P S} \sqrt{M_{P S}}$ become much more sensitive to the value of $\alpha_{s}$ i.e. to $q^{*}$. This can be seen in Fig. 3 where $f_{P S} \sqrt{M_{P S}}$ is plotted versus $1 / M_{P S}$ for $q^{*}$ $=1 / a$ and $q^{*}=\pi / a$. One sees that there is considerable dependence of the slope on $q^{*}$. We will quote a central value for the slope based on the $\beta=6.0$ data using $q^{*}=2 / a$ and allow for a change in $q^{*}$ in our error. We note that in order for the expansion in Eq. (21) to be valid there should be only power dependence and no logarithmic dependence on $M_{Q}$. We satisfy this condition by fixing the logarithms in the matching coefficients to $\log \left(a M_{0}^{b}\right)$. Our best estimate for $\Lambda_{s l}$

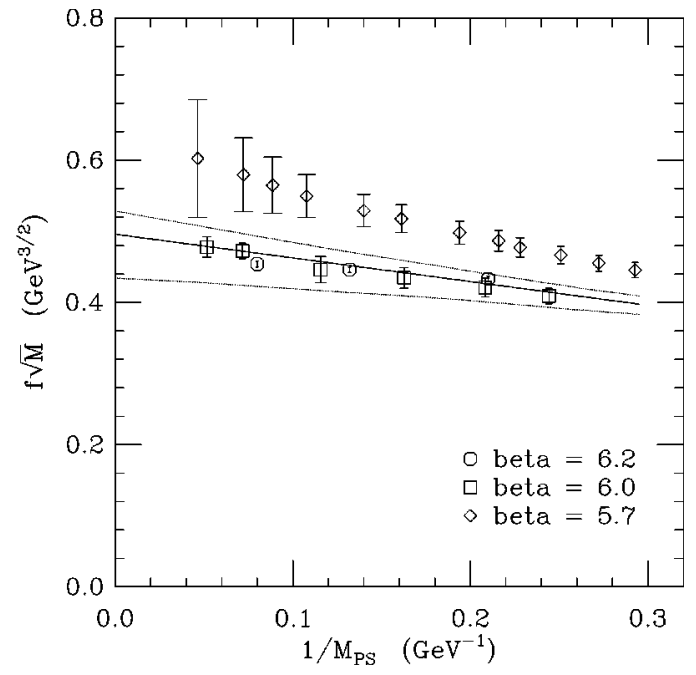

FIG. 4. Results for $f_{P S} \sqrt{M_{P S}}$ versus $1 / M_{P S}$ from different $\beta$ 's. $q^{*}=2 / a$ is used for the one-loop renormalized data points. The central full line is a linear fit to the $q^{*}=2 / a, \beta=6.0$ data. The bottom (top) dotted line comes from lower (upper) bounds on fits to $q^{*}=1 / a\left(q^{*}=\pi / a\right), \beta=6.0$ data (see text).

is then: $\Lambda_{s l}=-0.67(9)\left(\begin{array}{c}-17 \\ +34\end{array}\right)(12) \mathrm{GeV}$. The first error is the statistical fit error to the $q^{*}=2 / a$ data and the second comes from the upper limit of errors on the magnitude of the slope from fits to $q^{*}=\pi / a$ data and the lower limit of errors in fits to $q^{*}=1 / a$ data. The third error is an estimate for $\alpha_{s}^{2}$ and $\alpha_{s}^{2} /\left(a M_{Q}\right)$ corrections. In Fig. 4 we plot the $\beta=6.0$ results together with data from other $\beta$ values all using $q^{*}=2 / a$. One sees that slopes extracted at the two finer lattice spacings are consistent within our large errors. Results from $\beta$ $=5.7$ lie mostly parallel to the $\beta=6.0$ and $\beta=6.2$ data, however statistical errors are too large near the static limit to enable a useful estimate for the slope. Around the physical $B$ meson the $\beta=5.7$ data points lie $\sim 15 \%$ high, in agreement with results in Table VI and consistent with our estimates of systematic errors in Table III.

In contrast to $f_{B_{s}}$ which we demonstrated can be calculated with $\sim 10 \%$ errors, we find that specific $1 / M_{Q}$ corrections are harder to determine with comparable percentage accuracy. For the two quantities discussed in this section, the $1 / M_{Q}$ corrections coming from the current corrections and the slope of $f_{P S} \sqrt{M_{P S}}$ as one leaves the static limit, the main reason is uncertainty coming from higher order perturbative corrections.

\section{SIMULATIONS AT THE CHARM QUARK AND $f_{D_{s}}$}

In this section we discuss the behavior of the decay constant and the current matrix elements for heavy quark masses in the charm region. For the coarsest of our lattice spacings the charm quark can be reached at a bare mass of $a M_{0}^{c}$ $=0.87(6)(3)\left({ }_{-13}^{+0}\right)[15]$.

The size of the lattice current matrix elements for different values of the heavy quark mass is compared in Fig. 5. This figure is similar to Fig. 2, however, at $\beta=5.7$ we cover a much wider range of mass values. We also plot $1 / M_{Q}^{2}$ 


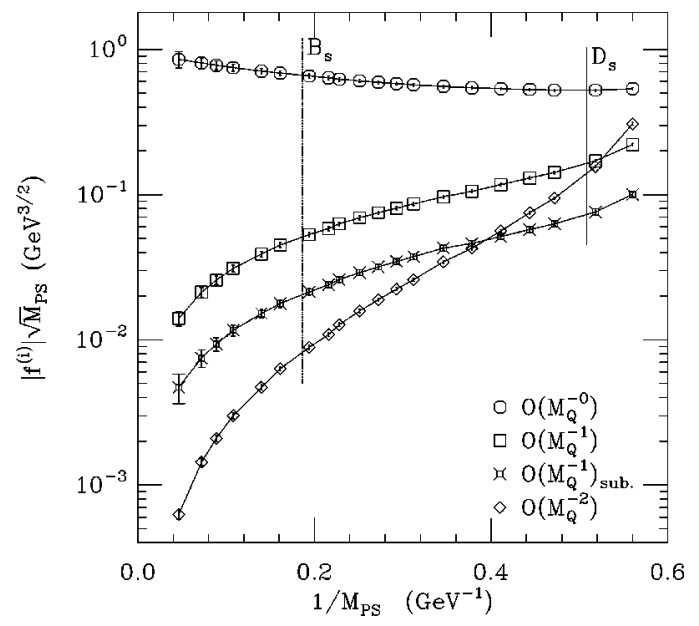

FIG. 5. Matrix elements $f^{(0)} \sqrt{M},\left|f^{(1)}\right| \sqrt{M},\left|f_{\text {sub }}^{(1)}\right| \sqrt{M}$, and $\left(\left|f^{(3)}+f^{(4)}+f^{(5)}\right|\right) \sqrt{M}$ versus $1 / M_{P S}$ at $\beta=5.7 . q^{*}=2 / a$ was used to obtain $\left|f_{\text {sub }}^{(1)}\right| \sqrt{M}$.

current matrix elements. The figure shows the value of $\left|f_{\text {sub }}^{(1)}\right| \sqrt{M}$ to be subleading even in the charm region, however it has grown from $4 \%$ of $f^{(0)} \sqrt{M}$ at the $B_{s}$ to $14 \%$ at the $D_{s}$. For $a M_{Q} \approx 1$ the value of the $1 / M_{Q}^{2}$ current matrix element $\left(\left|f^{(3)}+f^{(4)}+f^{(5)}\right|\right) \sqrt{M}$ is equal to $\left|f^{(1)}\right| \sqrt{M}$ and the two lines in Fig. 5 cross. After the discussion in the previous section, this is not unexpected and provides evidence that this current is also dominated by power law contributions. The part of the current $\left\langle J_{0}^{(3)}+J_{0}^{(4)}+J_{0}^{(5)}\right\rangle$ proportional to $\left\langle J_{0}^{(0)}\right\rangle$ diverges as $\alpha_{s} /\left(a M_{Q}\right)^{2}$. For $a M_{0} \approx 1$ this results in a suppression of $\mathcal{O}\left(\alpha_{s}\right)$ with respect to $\left\langle J_{0}^{(0)}\right\rangle$, which is the same suppression factor as the one for $\left\langle J_{0}^{(1)}\right\rangle$. In order to avoid uncertainties of $\mathcal{O}\left[\alpha_{s} /\left(a M_{Q}\right)^{2}\right]$ in the final $f_{D_{s}}$, we do not include the matrix elements of $1 / M_{Q}^{2}$ currents. By doing so, we have to accept an uncertainty of the size of $\Lambda_{\mathrm{QCD}}^{2} / M_{Q}^{2}$, which is $10 \%$, for the final result. This is a conservative estimate, however. Based on our experience with $\Lambda_{\mathrm{QCD}} / M_{Q}$ current corrections, we can expect that most of $\left(\left|f^{(3)}+f^{(4)}+f^{(5)}\right|\right) \sqrt{M}$ plotted in Fig. 5 will eventually be cancelled by matching coefficients at $O\left[\alpha_{s} /\left(a M_{Q}\right)^{2}\right]$. So, even at the $D_{s}$, the true $\Lambda_{\mathrm{OCD}}^{2} / M_{Q}^{2}$ current corrections should not be a large effect. The other $\Lambda_{\mathrm{QCD}}^{2} / M_{Q}^{2}$ contributions in $f_{D_{s}}$ come from the $\Lambda_{\mathrm{QCD}}^{2} / M_{Q}^{2}$ terms in the NRQCD action, and these are already included in the present simulations. To estimate the total NRQCD error we add to the $10 \%$ another $2 \%$ coming from $O\left(\alpha_{s} \Lambda_{\mathrm{QCD}} / M_{Q}\right)$ errors in the NRQCD action (see discussion at the end of Sec. II).

For the perturbative uncertainties we assign $11 \%$ for $\alpha_{s}^{2}$. This is larger than the uncertainty of $f_{D_{s}}$ arising from the variation of $q^{*}$ in a range from 1 to $\pi$. For the residual power law contributions of $\mathcal{O}\left(\alpha_{s}^{2} / a M_{Q}\right)$ we assign an uncertainty of $13 \%$, which leads to $17 \%$ for the total perturbative error, when added in quadrature. The $13 \%$ uncertainty is comparable to the size of the entire $\left\langle J_{0}^{(1)}\right\rangle_{\text {sub }}$ matrix element. For the uncertainty in the strange quark mass we assign $4 \%$ as in the case of $f_{B_{s}}$, which turns out to be negligible compared to the other sources of uncertainty. It is interesting to
TABLE VII. Simulation details for Sec. VI.

\begin{tabular}{lcc}
\hline \hline & $\beta=6.0$ & $\beta=6.2$ \\
\hline volume & $16^{3} \times 48$ & $24^{3} \times 48$ \\
No. configs & 268 & 144 \\
$c_{S W}$ & 1.77 (nonpert.) & 1.61 (nonpert.) \\
$a M_{0}$ & 2.0 & 1.44 \\
$\kappa_{q}$ & 0.13344 & 0.1346 \\
\hline \hline
\end{tabular}

note that the uncertainty arising from the scale $a\left(m_{\rho}\right)$ almost cancels between the effect this has on the conversion of the matrix element into physical units and the shift of the bare charm quark mass.

For our final result we obtain

$$
\begin{aligned}
f_{D_{s}} & =223(6)(31)(38)(23)(9)\left(\begin{array}{c}
+3 \\
-1
\end{array}\right) \mathrm{MeV} \\
& \rightarrow f_{D_{s}}=223(55) \mathrm{MeV}
\end{aligned}
$$

The uncertainties are listed in the same order as in Table III with the two discretization errors added in quadrature. This result is in very good agreement with the lattice average of $220_{-20}^{+25} \mathrm{MeV}$ quoted in Ref. [29] for quenched $f_{D_{s}}$, however, the uncertainty we assign is larger. It is also interesting to compare with direct experimental determinations of $f_{D_{s}}$. In a recent review article the authors of Ref. [30] present a world averaged experimental value of $f_{D_{s}}=(241$ $\pm 32) \mathrm{MeV}$, which is very consistent with the lattice numbers. Recall, however, that the lattice results given here are all in the quenched approximation.

\section{VI. $f_{B_{s}}$ FROM FINITE MOMENTUM MESONS}

This section investigates the defining matrix element Eq. (1) for nonzero spatial momentum $\vec{p}$ at two lattice spacings $\beta=6.0$ and $\beta=6.2$. Our goal is not to calculate $f_{B_{s}}$ more accurately than we have done in Sec. III from $B$ mesons at rest, but to test our ability to simulate hadrons at finite spatial momenta and obtain consistent results. At each $\beta$ we work at one value for the heavy quark mass and we do not attempt to extrapolate to the physical $b$ quark. Furthermore at $\beta=6.0$ we use a different set of configurations from the previous sections. The new configurations and light propagators were provided by the UKQCD Collaboration. In Table VII we summarize relevant action parameters.

We accumulated data for four nonzero momenta $(0,0,1)$, $(0,1,1),(1,1,1)$, and $(0,0,2)$ in units of $2 \pi / a L$ averaging over all equivalent momenta $\pm p_{x}, \pm p_{y}$, etc. From the difference of falloff energies of meson correlations with and without momenta

$$
\delta E(p) \equiv E_{\text {sim }}(p)-E_{\text {sim }}(0),
$$

one can use a relativistic dispersion relation to define a mass for the heavy meson, usually called $M_{\text {kin }}$,

$$
M_{\mathrm{kin}}=\left(p^{2}-\delta E^{2}\right) /(2 \delta E) .
$$


TABLE VIII. $a M_{\text {kin }}$ extracted from pseudoscalar mesons with different momenta and comparison with $a M_{\text {pert }}$. Momenta are given in units of $2 \pi / a L$. Values for the lowest momenta in lattice units are $a p=0.39(\beta=6.0)$ and $0.26(\beta=6.2)$.

\begin{tabular}{lll}
\hline \hline & $\beta=6.0$ & $\beta=6.2$ \\
$a M_{\text {pert }}$ & $2.53(8)$ & $1.88(5)$ \\
\hline$a M_{\text {kin }}$ & & \\
$(0,0,1)$ & $2.51(23)$ & $1.93(33)$ \\
$(0,1,1)$ & $2.83(36)$ & $1.77(19)$ \\
$(1,1,1)$ & & $1.73(17)$ \\
$(0,0,2)$ & $2.49(11)$ & $1.63(16)$ \\
\hline \hline
\end{tabular}

Our first test is to check the extent to which $M_{\text {kin }}$ is independent of $p$. This nonperturbatively determined mass can also be compared with another mass $M_{\text {pert }}$ based on the perturbative pole mass for the $b$ quark combined with an energy shift $E_{0}$ and simulation results for $E_{\text {sim }}(p=0)$

$$
M_{\text {pert }}=Z_{m} M_{0}-E_{0}+E_{\text {sim }}(0) .
$$

We use one-loop perturbation theory for $Z_{m}$ and $E_{0}$. The one-loop coefficients and $q^{*}$ for the combination $\left(Z_{m} M_{0}\right.$ $\left.-E_{0}\right)$ are given in Ref. [15]. In Table VIII we list results for $M_{\text {kin }}$ and $M_{\text {pert }}$ in lattice units. The errors in $M_{\text {kin }}$ are statistical and for $M_{\text {pert }}$ we give $O\left(\alpha_{s}^{2}\right)$ perturbative errors. The statistical errors in $M_{\text {pert }}$ coming from $E_{\text {sim }}(0)$ are negligible compared to the perturbative errors. The quality of our signals for finite momentum mesons depended rather strongly on the smearings employed (see Ref. [15] for details of smearings). In some instances, e.g. in the $\beta=6.0$ data, clearer signals were obtained for the highest momentum than for some of the lower ones. We believe this is because the smearings used were better suited for that momentum. For momentum $(1,1,1)$, on the other hand, we could not extract any useful results at $\beta=6.0$. Figure 6 plots $a M_{\text {kin }}$ versus the

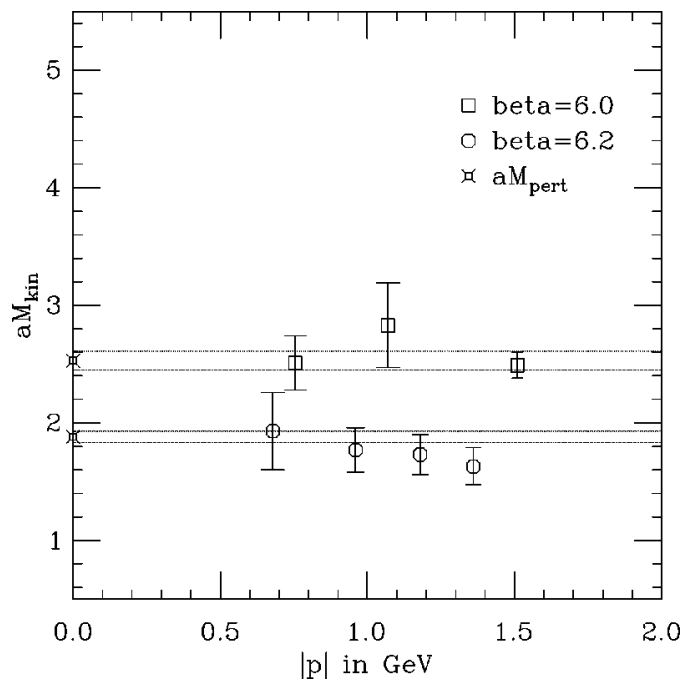

FIG. 6. $a M_{\text {kin }}$ extracted from mesons with different spatial momenta. The fancy squares to the left show $a M_{\text {pert }}$. The horizontal lines bracket perturbative errors on $a M_{\text {pert }}$.
TABLE IX. The ratios $R(p)$ and $R^{(0)}(p)$ of Eq. (27) and (28) for several momenta and $\beta$ values. Momenta are given in units of $2 \pi / a L$.

\begin{tabular}{lcccc}
\hline \hline & \multicolumn{2}{c}{$\beta=6.0$} & \multicolumn{2}{c}{$\beta=6.2$} \\
momentum & $R(p)$ & $R^{(0)}(p)$ & $R(p)$ & $R^{(0)}(p)$ \\
\hline$(0,0,1)$ & $1.02(2)$ & $1.01(2)$ & $1.01(2)$ & $1.00(2)$ \\
$(0,1,1)$ & $1.00(5)$ & $0.98(5)$ & $1.03(3)$ & $1.02(3)$ \\
$(1,1,1)$ & & & $1.05(4)$ & $1.03(4)$ \\
$(0,0,2)$ & $1.08(3)$ & $1.05(3)$ & $1.10(6)$ & $1.07(6)$ \\
\hline \hline
\end{tabular}

momentum and compares with $a M_{\text {pert }} . M_{\text {kin }}$ is reasonably independent of $p$ for the range considered and agrees with $M_{\text {pert }}$ within $\sim 1 \sigma$. The statistical errors at some of our data points, however, are still considerable for $M_{\text {kin }}$ and they could be hiding systematic discretization corrections.

The next quantity of interest is the ratio of the decay matrix element for a heavy meson with momentum $\vec{p}$ to that of a heavy meson decaying at rest [31]. More specifically we consider

$$
\frac{\left\langle 0\left|A_{0}\right| P S, \vec{p}\right\rangle / \sqrt{E(p)}}{\left\langle 0\left|A_{0}\right| P S, \vec{p}=0\right\rangle / \sqrt{M_{P S}}}=\frac{\sqrt{E(p)}}{\sqrt{M_{P S}}},
$$

where $E(p)$ is the total energy of the meson. The RHS of Eq. (26) is a very slowly rising function of $p$, never getting very much above 1 for the momenta involved. In our simulations we take the following expression for the LHS, working to the same order in $1 / M_{Q}$ as in the previous sections.

$$
R(p) \equiv \frac{\sum_{j=0}^{2} C_{j}^{\left(A_{0}\right)}\left\langle 0\left|J_{0}^{(j)}\right| \vec{p}\right\rangle / \sqrt{E(p)}}{\sum_{j=0}^{2} C_{j}^{\left(A_{0}\right)}\left\langle 0\left|J_{0}^{(j)}\right| \vec{p}=0\right\rangle / \sqrt{M_{P S}}}
$$

Whereas $M_{\text {kin }}$, discussed above, checks for the correct relativistic dispersion relation in the energies, $R(p)$ tests the $p$ dependence of amplitudes. In Table IX we give results for $R(p)$. We also list $R^{(0)}(p)$, the analogous ratio using only the zeroth order current $J_{0}^{(0)}$,

$$
R^{(0)}(p) \equiv \frac{\left\langle 0\left|J_{0}^{(0)}\right| \vec{p}\right\rangle / \sqrt{E(p)}}{\left\langle 0\left|J_{0}^{(0)}\right| \vec{p}=0\right\rangle / \sqrt{M_{P S}}} .
$$

One sees only a small change at less than the $1 \sigma$ level between $R(p)$ and $R^{(0)}(p)$. Most of the effect of one-loop matching and $1 / M_{Q}$ current corrections is canceled in this ratio. In Fig. 7 we plot $R(p)$ versus the momentum. The full line is the expected behavior coming from the RHS of Eq. (26). A single line suffices for the two $\beta$ values, since the difference in $M_{P S}$ is negligible. One sees that results consistent with continuum expectations are obtained up to momenta of about $1.2 \mathrm{GeV}$. Even for the largest momenta deviations are less than $\sim 8 \%$. 


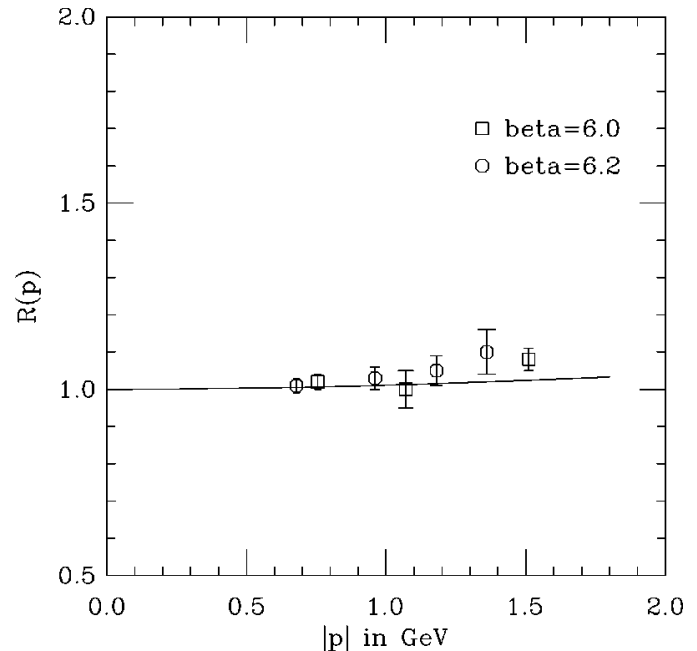

FIG. 7. The ratio $R(p)$ of Eq. (27) versus momentum. The full line shows $\sqrt{E(p)} / \sqrt{M_{P S}}$.

\section{SUMMARY}

In this article we presented further tests of $f_{B_{S}}$ determinations using NRQCD heavy quarks and clover light quarks. We checked for scaling, studied the dependence on the momentum of the decaying $B_{s}$ meson and also investigated cancellation of power law terms through $O\left[\alpha_{s} /(a M)\right]$. At one value of the lattice spacing we were able to determine the $D_{s}$ meson decay constant. Our best values for $f_{B_{s}}$ and $f_{D_{s}}$ are given in Eqs. (15) and (22), respectively. Our results are in good agreement with other lattice determinations of these quantities. In the case of $f_{D_{s}}$, for which experimental measurements exist, we obtain a value consistent with the current experimental world average as given in Ref. [30].

\section{ACKNOWLEDGMENTS}

This work was supported by the U.S. DOE under Grant No. DE-FG02-91ER40690, PPARC under Grant No. GR/ L56343, NATO under CRG/94259 and by NSF. Simulations were carried out at NERSC and at EPCC. We thank the UKQCD collaboration for providing many of the configurations and light propagators. S.C. acknowledges financial support from the Royal Society of Edinburgh. J.Sh. would like to thank members of the theoretical physics group at the University of Glasgow for their hospitality during an extended visit. Support from PPARC grant PPA/V/S/1997/ 00666 is gratefully acknowledged.
TABLE X. One-loop matching coefficients $\rho_{0}, \rho_{1}$ and $\rho_{2}$ of Eq. (17) and mixing coefficient $\zeta_{10}$ of Eq. (18) for several heavy quark mass values. $\rho_{2}$ includes $2 a M_{0} \zeta_{A_{0}}$ in order to incorporate contributions from $J^{\text {(disc) }}$. The NRQCD action is that of Eqs. (4)-(6) with $c_{i}=1$. Both the NRQCD and clover light quark actions are taken to be tadpole improved with the plaquette definition of $u_{0}$.

\begin{tabular}{|c|c|c|c|c|c|}
\hline$a M_{0}$ & $n$ & $\rho_{0}$ & $\rho_{1}$ & $\rho_{2}$ & $\zeta_{10}$ \\
\hline 0.600 & 10 & $0.1875(6)$ & $0.705(3)$ & $0.487(2)$ & $-0.9944(3)$ \\
\hline 0.800 & 8 & $-0.1191(4)$ & $0.213(2)$ & $0.758(2)$ & $-0.7968(2)$ \\
\hline 0.800 & 5 & $-0.1203(4)$ & $0.261(2)$ & $0.680(2)$ & $-0.7682(2)$ \\
\hline 1.000 & 6 & $-0.2207(3)$ & $0.026(2)$ & $0.914(2)$ & $-0.6645(1)$ \\
\hline 1.000 & 4 & $-0.2108(3)$ & $0.045(2)$ & $0.849(2)$ & $-0.6502(1)$ \\
\hline 1.125 & 6 & $-0.2546(3)$ & $-0.035(2)$ & $1.026(2)$ & $-0.6063(1)$ \\
\hline 1.200 & 3 & $-0.2500(3)$ & $-0.031(2)$ & $0.987(3)$ & $-0.5613(1)$ \\
\hline 1.300 & 5 & $-0.2814(3)$ & $-0.068(2)$ & $1.156(3)$ & $-0.5387(1)$ \\
\hline 1.400 & 3 & $-0.2806(3)$ & $-0.070(2)$ & $1.187(3)$ & $-0.5014(1)$ \\
\hline 1.440 & 3 & $-0.2855(3)$ & $-0.071(2)$ & $1.227(3)$ & $-0.4909(1)$ \\
\hline 1.500 & 4 & $-0.2987(3)$ & $-0.080(2)$ & $1.324(3)$ & $-0.4790(1)$ \\
\hline 1.500 & 2 & $-0.2732(3)$ & $-0.061(2)$ & 1.193(3) & $-0.4675(1)$ \\
\hline 1.600 & 2 & $-0.2845(2)$ & $-0.065(2)$ & $1.311(4)$ & $-0.4463(1)$ \\
\hline 1.700 & 2 & $-0.2941(2)$ & $-0.064(2)$ & $1.425(4)$ & $-0.4267(1)$ \\
\hline 2.000 & 2 & $-0.3146(2)$ & $-0.046(2)$ & $1.781(4)$ & $-0.3768(1)$ \\
\hline 2.500 & 3 & $-0.3382(2)$ & $-0.006(2)$ & $2.454(6)$ & $-0.31626(7)$ \\
\hline 2.500 & 2 & $-0.3331(2)$ & $-0.001(2)$ & $2.432(6)$ & $-0.31525(7)$ \\
\hline 2.700 & 2 & $-0.3374(2)$ & $0.018(2)$ & $2.706(6)$ & $-0.29599(6)$ \\
\hline 3.000 & 2 & $-0.3421(2)$ & $0.044(2)$ & $3.132(7)$ & $-0.27122(6)$ \\
\hline 3.500 & 2 & $-0.3457(2)$ & $0.094(2)$ & $3.895(8)$ & $-0.23818(6)$ \\
\hline 4.000 & 2 & $-0.3460(2)$ & $0.135(2)$ & $4.705(10)$ & $-0.21235(5)$ \\
\hline 4.000 & 1 & $-0.3374(2)$ & $0.140(2)$ & $4.600(10)$ & $-0.21147(5)$ \\
\hline 4.500 & 1 & $-0.3379(2)$ & $0.176(3)$ & $5.467(11)$ & $-0.19115(5)$ \\
\hline 5.000 & 1 & $-0.3362(2)$ & $0.211(3)$ & $6.328(12)$ & $-0.17433(4)$ \\
\hline 7.000 & 1 & $-0.3173(2)$ & $0.321(4)$ & $10.050(21)$ & $-0.12898(3)$ \\
\hline 10.000 & 1 & $-0.2770(2)$ & $0.432(6)$ & $15.957(30)$ & $-0.09282(3)$ \\
\hline 12.500 & 1 & $-0.2432(2)$ & $0.505(7)$ & $20.905(36)$ & $-0.07523(2)$ \\
\hline 20.000 & 1 & $-0.1528(2)$ & $0.655(12)$ & $36.335(64)$ & $-0.04797(1)$ \\
\hline
\end{tabular}

\section{APPENDIX: MATCHING COEFFICIENTS FOR $\boldsymbol{A}_{0}$}

In Table $\mathrm{X}$ we collect matching coefficients for $A_{0}$, i.e., the $\rho_{0}, \rho_{1}, \rho_{2}$ and the mixing coefficient $\zeta_{10}$ defined in Eqs. (17) and (18). This augments numbers given in Ref. [20] where, for the case of the full NRQCD action used in the present simulations, a smaller set of heavy quark masses was covered. The coefficient $\rho_{2}$ in Table X includes contributions from $J_{0}^{(\text {disc) }}$, as explained after Eq. (11). We note that in the first article of Ref. [20] a different convention was used and $\rho_{2}$ there did not include $2 a M_{0} \zeta_{A_{0}}$. The new convention adopted here is more in line with those employed in the second article of Ref. [20] for matching of $A_{k}, V_{0}$, and $V_{k}$.
[1] S. Hashimoto, review talk given at Lattice '99 [Nucl. Phys. B (Proc. Suppl.) 83-84, 3 (2000)].

[2] JLQCD Collaboration, S. Aoki et al., Phys. Rev. Lett. 80, 5711 (1998).
[3] A. Ali Khan et al., Phys. Lett. B 427, 132 (1998).

[4] JLQCD Collaboration, K-I. Ishikawa et al., Phys. Rev. D 61, 074501 (2000).

[5] A.X. El-Khadra et al., Phys. Rev. D 58, 014506 (1998). 
[6] MILC Collaboration, C. Bernard et al., Phys. Rev. Lett. 81, 4812 (1998).

[7] APE Collaboration, D. Becirevic et al., Phys. Rev. D 60, 074501 (1999).

[8] UKQCD Collaboration, C. Maynard et al., Nucl. Phys. Proc. B Suppl. 83-84, 322 (2000).

[9] S. Collins et al., Phys. Rev. D 60, 074504 (1999).

[10] CP-PACS Collaboration, A. Ali Khan et al., Nucl. Phys. B (Proc. Suppl.) 83-84, 265 (2000).

[11] CP-PACS Collaboration, H. Shanahan et al., Nucl. Phys. B (Proc. Suppl.) 83-84, 331 (2000).

[12] G.P. Lepage et al., Phys. Rev. D 46, 4052 (1992).

[13] A. El-Khadra, A. Kronfeld, and P. Mackenzie, Phys. Rev. D 55, 3933 (1997).

[14] UKQCD Collaboration, H. Shanahan et al., Phys. Rev. D 55, 1548 (1997).

[15] J. Hein et al., Phys. Rev. D 62, 074503 (2000).

[16] P.A. Rowland, Ph.D. thesis, University of Edinburgh, 1997.

[17] M. Göckeler et al., Phys. Rev. D 57, 5562 (1998).

[18] J. Hein et al., Nucl. Phys. B (Proc. Suppl.) 73, 366 (1999).

[19] S. Collins et al., Nucl. Phys. B (Proc. Suppl.) 83-84, 271
(2000).

[20] C. Morningstar and J. Shigemitsu, Phys. Rev. D 57, 6741 (1998); 59, 094504 (1999).

[21] S. Collins et al. (in preparation).

[22] G.P. Lepage and P.B. Mackenzie, Phys. Rev. D 48, 2250 (1993).

[23] K. Jansen et al., Phys. Lett. B 372, 275 (1996).

[24] C.T.H. Davies et al., Phys. Lett. B 345, 42 (1995).

[25] S.J. Brodsky, G.P. Lepage, and P.B. Mackenzie, Phys. Rev. D 28, 228 (1983).

[26] O. Hernandez and B. Hill, Phys. Rev. D 50, 495 (1994).

[27] C. Bernard, M. Golterman, and C. McNeile, Phys. Rev. D 59, 074506 (1999).

[28] M. Crisafulli, V. Lubicz, and A. Vladikas, Eur. Phys. J. C 4, 145 (1998).

[29] T. Draper, review talk given at Lattice '98 [Nucl. Phys. B (Proc. Suppl.) 73, 43 (1999)].

[30] F. Parodi, P. Roudeau, and A. Stocchi, Nuovo Cimento A 112, 833 (1999).

[31] Similar studies are reported in J.N. Simone, Nucl. Phys. B (Proc. Suppl.) 53, 386 (1997). 